\title{
A new subclasses of meromorphic univalent functions associated with a differential operator
}

\author{
Hazha Zirar Hussain \\ Department of Mathematics, College of Science, University of Salahaddin, \\ Erbil,Iraq. \\ E-mail : hazhazirar@yahoo.com
}

Recived : $1111 \backslash 2018$

Revised : $11 \backslash 11 \backslash 2018$

Accepted : 27\1112018

Available online : $24 / 1 / 2019$

DOI: 10.29304/jqcm.2019.11.1.460

\begin{abstract}
In this paper we have introduced and studied some new subclasses of meromorphic univalent functions which are defined by means of a differential operator. We have obtained numerous sharp results including coefficient conditions, extreme points, distortion bounds and convex combinations for the above classes of meromorphic univalent functions.
\end{abstract}

Keywords: Univalent Functions, Meromorphic Functions, Differential Operator, Distortion Inequality, Extreme Points.

Mathematics Subject Classification:64S40. 


\section{Introduction}

Let $\mathfrak{H}$ denote the class of functions which are analytic in the punctured disk $\mathcal{U}^{*}=\{z: 0<|z|<$ $1\}$ of the form

$$
f(z)=\frac{a_{0}}{z}+\sum_{n=1}^{\infty} a_{n} z^{n}, a_{0}>0 .
$$

Suppose that $\mathfrak{H}^{*}$ denote the subclass of $\mathfrak{H}$ consisting of functions that are univalent in $\mathcal{U}^{*}$.

Further $\mathfrak{S}_{m}^{*}$ denote subclass of $\mathfrak{H}^{*}$ consisting of functions $f$ of the form

$$
f(z)=\frac{a_{0}}{z}+\sum_{n=0}^{\infty} a_{m+n} z^{m+n}, \quad a_{0}>0, a_{m+n}
$$

Definition: A function $f \in \mathfrak{H}_{m}^{*}$ is said to be meromorphic starlike of order $\alpha$ in $\mathcal{U}^{*}$ if it satisfies the inequality

$$
\operatorname{Re}\left\{\frac{z f^{\prime}(z)}{f(z)}\right\}>-\alpha, z \in \mathcal{U}^{*}, 0 \leq \alpha<1 .
$$

On the other hand, a function $f \in \mathfrak{S}_{m}^{*}$ is said to be meromorphic convex of order $\alpha$ in $\mathcal{U}^{*}$ if it satisfies the inequality

$$
\begin{aligned}
\operatorname{Re}\left\{1+\frac{z f^{\prime \prime}(z)}{f^{\prime}(z)}\right\} & >-\alpha, z \in \mathcal{U}^{*}, 0 \leq \alpha \\
& <1 .
\end{aligned}
$$

Various subclasses of $\mathfrak{H}$ have been introduced and studied by many authors see [1], [2], [5], [7], [8], [16],[17],[19], [20], [21] and [23] In recent years, some subclasses of meromorphic functions associated with several families of integral operators and derivative operators were introduced and investigated see [7] [8], [18] and [4],[15]. The first differential operator for meromorphic function was introduced by Fraisin and Darus [10]. Ghanim and Darus introduced a differential operator [11]:

$$
\begin{gathered}
I^{0} f(z)=f(z), \\
I^{1} f(z)=z f^{\prime}(z)+\frac{2 a_{0}}{z}, \\
I^{2} f(z)=z\left(I^{1} f(z)\right)^{\prime}+\frac{2 a_{0}}{z}, \\
I^{k} f(z)=z\left(I^{(k-1)} f(z)\right)^{\prime}+\frac{2 a_{0}}{z},
\end{gathered}
$$

where $k \in \mathbb{N}_{0}=\mathbb{N} \bigcup\{0\}, z \in \mathcal{U}^{*}$.

For a function $f$ in $\mathfrak{H}_{m}^{*}$, from definition of the differential operator $I^{k} f(z)$, we easily see that

$$
\begin{aligned}
I^{k} f(z)=\frac{a_{0}}{z}+ & \sum_{n=0}^{\infty} n^{k} a_{m+n} z^{m+n}, \quad a_{0}>0, a_{m+n} \\
& >0, m \in \mathbb{N}, k \in \mathbb{N}_{0}=\mathbb{N} \bigcup\{0\}, z \\
& \in \mathcal{U}^{*} . \quad \text { (1.5) }
\end{aligned}
$$

By using the operator $I^{k}$, some authors have established many subclasses of meromorphic functions, for example [9], [11],[12] and [13]. With the help of the differential operator $I^{k}$, we define the following new class of meromorphic univalent functions and obtain some interesting results.

Let $\mathfrak{H}_{m, k}^{*}(\eta, \theta, \vartheta)$, denote the family of meromorphic univalent functions $f$ of the form (1.2) such that

$$
\left|\frac{z^{2}\left(I^{k} f(z)\right)^{\prime}+a_{0}}{\vartheta z^{2}\left(I^{k} f(z)\right)^{\prime}-a_{0}+(1+\vartheta) \eta a_{0}}\right|<\theta,
$$

For $0 \leq \eta<1,0<\theta \leq 1, \quad 0 \leq \vartheta \leq 1, k \in \mathbb{N}_{0}=$ $\mathbb{N} \cup\{0\}$, and $z \in \mathcal{U}^{*}$.

For a given real number $z_{0}\left(0<z_{0}<1\right)$. Let $\mathfrak{H}_{m i}(i=0,1)$ be a subclass of $\mathfrak{H}_{m}^{*}$ satisfying the condition $z_{0} f\left(z_{0}\right)=1$ and $-z_{0}{ }^{2} f^{\prime}\left(z_{0}\right)=1$ respectively.

Let

$$
\begin{aligned}
\mathfrak{S}_{m i, k}^{*}\left(\eta, \theta, \vartheta, z_{0}\right) & =\mathfrak{S}_{m, k}^{*}(\eta, \theta, \vartheta) \\
& \cap \mathfrak{H}_{m i},(i=0,1) .
\end{aligned}
$$

For other subclasses of meromorphic univalent functions, one may refer to the recent work of Aouf [2], Aouf and Darwish [3], Cho et al [8], Joshi et al [14], Srivastava and Owa [21] and [22]. Also we prove a necessary and sufficient condition for a subset $C$ of the real interval $[0,1]$ should satisfy the property $\quad \cup_{z_{r} \in C} \mathfrak{H}_{m 0, k}^{*}\left(\eta, \theta, \vartheta, z_{r}\right) \quad$ and $\cup_{z_{r} \in C} \mathfrak{H}_{m 1, k}^{*}\left(\eta, \theta, \vartheta, z_{r}\right)$ each constitute a convex family.

\section{Coefficient Inequalities}

In this section, we provide a necessary and sufficient condition for a function $f$ meromorphic univalent in $\mathcal{U}^{*} \quad$ to be in $\mathfrak{H}_{m, k}^{*}(\eta, \theta, \vartheta), \mathfrak{H}_{m 0, k}^{*}\left(\eta, \theta, \vartheta, z_{0}\right)$ and $\mathfrak{S}_{m 1, k}^{*}\left(\eta, \theta, \vartheta, z_{0}\right)$.

Theorem 2.1: A function $f(z) \in \mathfrak{H}_{m}^{*}$ defined by equation (1.2) is in the class $\mathfrak{S}_{m, k}^{*}(\eta, \theta, \vartheta)$ if and only if

$\sum_{n=0}^{\infty} n^{k}(m+n)(1+\vartheta \theta) a_{m+n} \leq \theta a_{0}(1-$

$\eta)(1+\vartheta), \quad(2.1)$

where $\quad 0 \leq \eta<1,0<\theta \leq 1, \quad 0 \leq \vartheta \leq 1, k \in$ $\mathbb{N}_{0}=\mathbb{N} U\{0\}$, and $z \in \mathcal{U}^{*}$. 
Hazha .Z

The result is sharp for the function given by

$$
\begin{gathered}
f(z)=\frac{a_{0}}{z}+\frac{\theta a_{0}(1-\eta)(1+\vartheta)}{n^{k}(m+n)(1+\vartheta \theta)} z^{m+n}, \quad n \\
\geq 1 \quad(2.2)
\end{gathered}
$$

Proof: Assume that the condition (2.1) is true. We must show that $f \in \mathfrak{H}_{m, k}^{*}(\eta, \theta, \vartheta)$ or equivalently prove that

$$
\begin{gathered}
\left|\frac{z^{2}\left(I^{k} f(z)\right)^{\prime}+a_{0}}{\vartheta z^{2}\left(I^{k} f(z)\right)^{\prime}-a_{0}+(1+\vartheta) \eta a_{0}}\right|<\theta, \\
\left|\frac{z^{2}\left(I^{k} f(z)\right)^{\prime}+a_{0}}{\vartheta z^{2}\left(I^{k} f(z)\right)^{\prime}-a_{0}+(1+\vartheta) \eta a_{0}}\right|= \\
\left|\frac{a_{0}+\left(-a_{0}+\sum_{n=1}^{\infty}(m+n) n^{k} a_{m+n} z^{m+n+1}\right)}{\vartheta\left(-a_{0}+\sum_{n=1}^{\infty}(m+n) n^{k} a_{m+n} z^{m+n+1}\right)-a_{0}+(1+\vartheta) \eta a_{0}}\right| \\
=\frac{\sum_{n=0}^{\infty}(m+n) n^{k} a_{m+n} z^{m+n+1}}{\vartheta\left(-a_{0}+\sum_{n=0}^{\infty}(m+n) n^{k} a_{m+n} z^{m+n+1}\right)-a_{0}+(1+\vartheta) \eta a_{0}} \mid \\
\leq \\
\left|\frac{\sum_{n=0}^{\infty}(m+n) n^{k} a_{m+n}}{\vartheta\left(-a_{0}+\sum_{n=0}^{\infty}(m+n) n^{k} a_{m+n}\right)-a_{0}+(1+\vartheta) \eta a_{0}}\right|<\theta .
\end{gathered}
$$

The last inequality is true by (2.1).

Conversely, suppose that $f \in \mathfrak{H}_{m, k}^{*}(\eta, \theta, \vartheta)$. We must show that the condition (2.1) holds true. We have

$$
\left|\frac{z^{2}\left(I^{k} f(z)\right)^{\prime}+a_{0}}{\vartheta z^{2}\left(I^{k} f(z)\right)^{\prime}-a_{0}+(1+\vartheta) \eta a_{0}}\right|<\theta .
$$

Thus

$$
\left|\frac{\sum_{n=0}^{\infty}(m+n) n^{k} a_{m+n} z^{m+n+1}}{\vartheta\left(-a_{0}+\sum_{n=0}^{\infty}(m+n) n^{k} a_{m+n} z^{m+n+1}\right)-a_{0}+(1+\vartheta) \eta a_{0}}\right|
$$

Since $\operatorname{Re}(z)<|z|$ for all $z$, we have

$$
\operatorname{Re}\left\{\frac{\sum_{n=0}^{\infty}(m+n) n^{k} a_{m+n} z^{m+n+1}}{\vartheta\left(-a_{0}+\sum_{n=0}^{\infty}(m+n) n^{k} a_{m+n} z^{m+n+1}\right)-a_{0}+(1+\vartheta) \eta a_{0}}\right\}
$$
$<\theta$.

Now, choosing values of $z$ on the real axise and allowing $z \rightarrow 1$ from the left through real values, the last inequality immediately yields the desired condition in (2.1).

Finally, it is observed that the result is sharp for the function given by

$f(z)=\frac{a_{0}}{z}+\frac{\theta a_{0}(1-\eta)(1+\vartheta)}{n^{k}(m+n)(1+\vartheta \theta)} z^{m+n}, \quad n \geq 1$.

Theorem 2.2: A function $f(z) \in \mathfrak{H}_{m}^{*}$ defined by equation (1.2) is in the class $\mathfrak{H}_{m 0, k}^{*}\left(\eta, \theta, \vartheta, z_{0}\right)$ if and only if

$$
\begin{gathered}
\sum_{n=0}^{\infty}\left[\frac{n^{k}(m+n)(1+\vartheta \theta)}{\theta(1-\eta)(1+\vartheta)}+\right. \\
\left.z_{0}{ }^{m+n+1}\right] a_{m+n} \leq 1,
\end{gathered}
$$

where $\quad 0 \leq \eta<1,0<\theta \leq 1, \quad 0 \leq \vartheta \leq 1, k \in$ $\mathbb{N}_{0}=\mathbb{N} U\{0\}$, and $z \in \mathcal{U}^{*}$.

The result is sharp for the function given by

$$
\begin{gathered}
f(z)=\frac{n^{k}(m+n)(1+\vartheta \theta)+\theta(1-\eta)(1+\vartheta) z^{m+n+1}}{z\left[n^{k}(m+n)(1+\vartheta \theta)+\theta(1-\eta)(1+\vartheta) z_{0}{ }^{m+n+1}\right]}, \\
m \in \mathbb{N}, n \geq 1 \quad(2.4)
\end{gathered}
$$

Proof: Assume that $f \in \mathfrak{H}_{m 0, k}^{*}\left(\eta, \theta, \vartheta, z_{0}\right)$, then

$$
\begin{gathered}
f\left(z_{0}\right)=\frac{a_{0}}{z_{0}}+\sum_{n=0}^{\infty} a_{m+n} z_{0}{ }^{m+n}, \quad a_{0}>0, a_{m+n} \\
z_{0} f\left(z_{0}\right)=a_{0}+\sum_{n=0}^{\infty} a_{m+n} z_{0}{ }^{m+n+1}, \quad a_{0}>0, a_{m+n} \\
>0, m \in \mathbb{N} \\
1=a_{0}+\sum_{n=0}^{\infty} a_{m+n} z_{0}{ }^{m+n+1}, a_{0}>0, a_{m+n} \\
a_{0}=1-\sum_{n=0}^{\infty} a_{m+n} z_{0}{ }^{m+n+1}, \quad a_{0}>0, a_{m+n}
\end{gathered}
$$

Subistituting equation (2.5) in inequality (2.1), we get

$$
\begin{gathered}
\sum_{n=0}^{\infty} n^{k}(m+n)(1+\vartheta \theta) a_{m+n} \\
\leq \theta\left(1-\sum_{n=0}^{\infty} a_{m+n} z_{0}^{m+n+1}\right)(1 \\
-\eta)(1+\vartheta) \\
\sum_{n=0}^{\infty} n^{k}(m+n)(1+\vartheta \theta) a_{m+n} \\
+\sum_{n=0}^{\infty} \theta(1-\eta)(1 \\
+\vartheta) a_{m+n} z_{0}^{m+n+1} \\
\leq \theta(1-\eta)(1+\vartheta)
\end{gathered}
$$

Thus,

$\sum_{n=0}^{\infty}\left[\frac{n^{k}(m+n)(1+\vartheta \theta)}{\theta(1-\eta)(1+\vartheta)}+z_{0}{ }^{m+n+1}\right] a_{m+n} \leq 1$.

Hence the proof is complete. 
Theorem 2.3: A function $f(z) \in \mathfrak{H}_{m}^{*}$ defined by equation (1.2) is in the class $\mathfrak{H}_{m 1, k}^{*}\left(\eta, \theta, \vartheta, z_{0}\right)$ if and only if

$\sum_{n=0}^{\infty}(m+n)\left[\frac{n^{k}(1+\vartheta \theta)}{\theta(1-\eta)(1+\vartheta)}-\right.$

$\left.z_{0}{ }^{m+n+1}\right] a_{m+n} \leq 1$,

where $\quad 0 \leq \eta<1,0<\theta \leq 1, \quad 0 \leq \vartheta \leq 1, k \in$ $\mathbb{N}_{0}=\mathbb{N} \cup\{0\}$, and $z \in \mathcal{U}^{*}$.

The result is sharp for the function given by $f(z)$

$=\frac{n^{k}(m+n)(1+\vartheta \theta)+\theta(1-\eta)(1+\vartheta) z^{m+n+1}}{z(m+n)\left[n^{k}(1+\vartheta \theta)-\theta(1-\eta)(1+\vartheta) z_{0}{ }^{m+n+1}\right]}, \quad m$ $\in \mathbb{N}, n \geq 1 \quad$ (2.7)

Proof: Assume that $f \in \mathfrak{H}_{m 1, k}^{*}\left(\eta, \theta, \vartheta, z_{0}\right)$, then

$$
\begin{gathered}
f\left(z_{0}\right)=\frac{a_{0}}{z_{0}}+\sum_{n=0}^{\infty} a_{m+n} z_{0}{ }^{m+n}, \quad a_{0}>0, a_{m+n} \\
>0, m \in \mathbb{N} \\
-z_{0}^{2} f^{\prime\left(z_{0}\right)}=a_{0}+\sum_{n=0}^{\infty}(m+n) a_{m+n} z_{0}{ }^{m+n+1}, a_{0} \\
>0, a_{m+n}>0, m \in \mathbb{N} \\
1=a_{0}+\sum_{n=0}^{\infty}(m+n) a_{m+n} z_{0}{ }^{m+n+1}, a_{0} \\
>0, a_{m+n}>0, m \in \mathbb{N} \\
a_{0}=1-\sum_{n=0}^{\infty} \begin{array}{c}
(m+n) a_{m+n} z_{0}{ }^{m+n+1}, a_{0} \\
>0, a_{m+n}>0, m \\
\in \mathbb{N}, \quad(2.8)
\end{array}
\end{gathered}
$$

subistituting equation (2.8) in equation (2.1), we get

$$
\begin{gathered}
\sum_{n=0}^{\infty} n^{k}(m+n)(1+\vartheta \theta) a_{m+n} \\
\leq \theta\left(1-\sum_{n=0}^{\infty}(m+n) a_{m+n} z_{0}^{m+n+1}\right)(1-\eta)(1 \\
+\vartheta)
\end{gathered}
$$

and,

$$
\begin{aligned}
& \sum_{n=0}^{\infty} n^{k}(m+n)(1+\vartheta \theta) a_{m+n} \\
&+\sum_{n=0}^{\infty} \theta(m+n)(1-\eta)(1 \\
&+\vartheta) a_{m+n} z_{0}{ }^{m+n+1} \\
& \leq \theta(1-)(1-\eta)(1+\vartheta)
\end{aligned}
$$

Thus,

$$
\sum_{n=0}^{\infty}(m+n)\left[\frac{n^{k}(1+\vartheta \theta)}{\theta(1-\eta)(1+\vartheta)}-z_{0}{ }^{m+n+1}\right] a_{m+n} \leq 1 .
$$

Hence the proof is complete.
From Theorem 2.2 and Theorem 2.3, we have the following results:

Corollary 2.1: If a function $f(z) \in \mathfrak{H}_{m}^{*}$ defined by (1.2) is in the class $\mathfrak{H}_{m 0, k}^{*}\left(\eta, \theta, \vartheta, z_{0}\right)$, then

$$
\begin{aligned}
& a_{m+n} \leq \\
& \frac{\theta(1-\eta)(1+\vartheta)}{n^{k}(m+n)(1+\vartheta \theta)+\theta(1-\eta)(1+\vartheta) z_{0}}{ }^{m+n+1}
\end{aligned}
$$

where $0 \leq \eta<1,0<\theta \leq 10 \leq \vartheta \leq 1, k \in \mathbb{N}_{0}=$ $\mathbb{N} \cup\{0\}$, and $z \in \mathcal{U}^{*}$.

Corollary 2.2: If a function $f(z) \in \mathfrak{H}_{m}^{*}$ defined by (1.2) is in the class $\mathfrak{H}_{m 1, k}^{*}\left(\eta, \theta, \vartheta, z_{0}\right)$, then

$a_{m+n} \leq$

$\frac{\theta(1-\eta)(1+\vartheta)}{(m+n)\left[n^{k}(1+\vartheta \theta)-\theta(1-\eta)(1+\vartheta) z_{0}{ }^{m+n+1}\right]}$,

where $0 \leq \eta<1,0<\theta \leq 10 \leq \vartheta \leq 1, k \in \mathbb{N}_{0}=$ $\mathbb{N} \cup\{0\}$, and $z \in \mathcal{U}^{*}$.

\section{Covering theorems}

In this section, distortion theorems will be considered and covering property for functions in the classes $\mathfrak{S}_{m 0, k}^{*}\left(\eta, \theta, \vartheta, z_{0}\right)$ and $\mathfrak{H}_{m 1, k}^{*}\left(\eta, \theta, \vartheta, z_{0}\right)$ will also be given.

Theorem 3.1: If a function $f(z) \in \mathfrak{S}_{m}^{*}$ defined by equation (1.2) is in the class $\mathfrak{S}_{m 0, k}^{*}\left(\eta, \theta, \vartheta, z_{0}\right)$, then

$$
\begin{aligned}
& |f(z)| \\
& \geq \frac{m(1+\vartheta \theta)-\theta(1-\eta)(1+\vartheta) r^{m+1}}{r\left[m(1+\vartheta \theta)+\theta(1-\eta)(1+\vartheta) z_{0}{ }^{m+1}\right]},
\end{aligned}
$$

where $0 \leq \eta<1,0<\theta \leq 10 \leq \vartheta \leq 1, k \in \mathbb{N}_{0}=$ $\mathbb{N} \cup\{0\}$, and $0<|z|<1$.

The result is sharp with the extremal function $f$ given by

$$
f(z)=\frac{m(1+\vartheta \theta)+\theta(1-\eta)(1+\vartheta) r^{m+1}}{r\left[m(1+\vartheta \theta)+\theta(1-\eta)(1+\vartheta) z_{0}{ }^{m+1}\right]} .
$$

Proof: Since $f \in \mathfrak{S}_{m 0, k}^{*}\left(\eta, \theta, \vartheta, z_{0}\right)$, by Theorem 2.2 we have

$$
\begin{aligned}
& m(1+\vartheta \theta)+\theta(1-\eta)(1+ \\
& \vartheta) z_{0}{ }^{m+1} \sum_{n=0}^{\infty} a_{m+n} \leq \sum_{n=0}^{\infty} n^{k}(m+n)(1+ \\
& \vartheta \theta)+\theta(1-\eta)(1+\vartheta) z_{0}{ }^{m+n+1} a_{m+n} \leq \\
& \theta(1-\eta)(1+\vartheta), \\
& \quad \sum_{n=0}^{\infty} a_{m+n} \\
& \quad \leq \frac{\theta(1-\eta)(1+\vartheta)}{m(1+\vartheta \theta)+\theta(1-\eta)(1+\vartheta) z_{0}{ }^{m+1}},
\end{aligned}
$$


Hazha .Z

Also we have

$$
\begin{gathered}
a_{0}=1-\sum_{n=0}^{\infty} a_{m+n} z_{0}^{m+n+1}, \quad a_{0}>0, a_{m+n} \\
>0, m \in \mathbb{N} \\
\geq \frac{m(1+\theta \vartheta)}{m(1+\vartheta \theta)+\theta(1-\eta)(1+\vartheta) z_{0}{ }^{m+1}},
\end{gathered}
$$

Thus from the above equation we obtain

$$
\begin{gathered}
|f(z)|=\left|\frac{a_{0}}{z}+\sum_{\substack{n=0 \\
>0, m \in \mathbb{N}}}^{\infty} a_{m+n} z^{m+n}\right|, a_{0}>0, a_{m+n} \\
\geq \frac{a_{0}}{r}-r^{m} \sum_{n=0}^{\infty} a_{m+n} \\
\geq \frac{m(1+\vartheta \theta)-\theta(1-\eta)(1+\vartheta) r^{m+1}}{r\left[m(1+\vartheta \theta)+\theta(1-\eta)(1+\vartheta) z_{0}{ }^{m+1}\right]} .
\end{gathered}
$$

Hence the proof is complete.

Theorem 3.2: If a function $f(z) \in \mathfrak{H}_{m}^{*}$ defined by equation (1.2) is in the class $\mathfrak{H}_{m 1, k}^{*}\left(\eta, \theta, \vartheta, z_{0}\right)$, then

$$
\begin{aligned}
& |f(z)| \\
& \leq \frac{m(1+\vartheta \theta)+\theta(1-\eta)(1+\vartheta) r^{m+1}}{r\left[m(1+\vartheta \theta)+\theta(1-\eta)(1+\vartheta) z_{0}{ }^{m+1}\right]}
\end{aligned}
$$

where $0 \leq \eta<1,0<\theta \leq 10 \leq \vartheta \leq 1, k \in \mathbb{N}_{0}=$ $\mathbb{N} \cup\{0\}$, and $0<|z|=r<1$.

The result is sharp with the extremal function $f$ given by

$$
f(z)=\frac{m(1+\vartheta \theta)+\theta(1-\eta)(1+\vartheta) r^{m+1}}{r m\left[(1+\vartheta \theta)+\theta(1-\eta)(1+\vartheta) z_{0}{ }^{m+1}\right]} .
$$

Proof: Since $f \in \mathfrak{H}_{m 1, k}^{*}\left(\eta, \theta, \vartheta, z_{0}\right)$ by Theorem 2.3 we have

$$
\begin{aligned}
& m(1+\vartheta \theta)+\theta(1-\eta)(1+ \\
& \vartheta) z_{0}{ }^{m+1} \sum_{n=0}^{\infty} a_{m+n} \leq \sum_{n=0}^{\infty} n^{k}(m+n)(1+ \\
& \vartheta \theta)+\theta(1-\eta)(1+\vartheta) z_{0}{ }^{m+n+1} a_{m+n} \leq \\
& \theta(1-\eta)(1+\vartheta), \\
& \quad \sum_{n=0}^{\infty} a_{m+n} \\
& \quad \leq \frac{\theta(1-\eta)(1+\vartheta)}{m(1+\vartheta \theta)-\theta(1-\eta)(1+\vartheta) z_{0}{ }^{m+1}} .
\end{aligned}
$$

Also we have

$$
\begin{gathered}
a_{0}=1+\sum_{n=0}^{\infty}(m+n) a_{m+n} z_{0}{ }^{m+n+1}, \quad a_{0} \\
>0, a_{m+n}>0, m \in \mathbb{N}, \\
\leq \frac{(1+\theta \vartheta)}{(1+\vartheta \theta)+\theta(1-\eta)(1+\vartheta) z_{0}{ }^{m+1}} .
\end{gathered}
$$

Thus from the above equation we obtain

$$
\begin{gathered}
|f(z)|=\left|\frac{a_{0}}{z}+\sum_{\substack{n=0 \\
>0, m \in \mathbb{N}}}^{\infty} a_{m+n} z^{m+n}\right|, a_{0}>0, a_{m+n} \\
\leq \frac{a_{0}}{r}+r^{m} \sum_{n=0}^{\infty} a_{m+n} \\
\leq \frac{m(1+\vartheta \theta)+\theta(1-\eta)(1+\vartheta) r^{m+1}}{r m\left[(1+\vartheta \theta)+\theta(1-\eta)(1+\vartheta) z_{0}{ }^{m+1}\right]} .
\end{gathered}
$$

Hence the proof is complete.

Corollary 3.1: The disk $0<|z|<1$ is mapped onto a domain that contains the disk $|w|<$ $\frac{m(1+\vartheta \theta)-\theta(1-\eta)(1+\vartheta) r^{m+1}}{\left[m(1+\vartheta \theta)+\theta(1-\eta)(1+\vartheta) z_{0}{ }^{m+1}\right]} \quad$ by any function $f \in \mathfrak{H}_{m 0, k}^{*}\left(\eta, \theta, \vartheta, z_{0}\right)$.

\section{Extreme Points}

The extreme points of the class $\mathfrak{H}_{m 0, k}^{*}\left(\eta, \theta, \vartheta, z_{0}\right)$ and $\mathfrak{H}_{m 1, k}^{*}\left(\eta, \theta, \vartheta, z_{0}\right)$ are given by the following theorem.

Theorem 4.1: Let $f_{0}(z)=\frac{1}{z}$

and

$$
\begin{aligned}
& f_{m+n}(z) \\
& =\frac{n^{k}(m+n)(1+\vartheta \theta)+\theta(1-\eta)(1+\vartheta) z^{m+n+1}}{z\left[n^{k}(m+n)(1+\vartheta \theta)+\theta(1-\eta)(1+\vartheta) z_{0}{ }^{m+n+1}\right]}, \\
& n \geq 0
\end{aligned}
$$

then $f(z)$ is in the class $\mathfrak{H}_{m 0, k}^{*}\left(\eta, \theta, \vartheta, z_{0}\right)$, if and only if it can be expressed in the form $f(z)=$ $\sum_{n=0}^{\infty} \gamma_{n} f_{m+n}(z) \quad$ where $\quad \gamma_{n} \geq 0, \gamma_{i}=0(i=$ $1,2, \ldots, m-1, m \geq 2$ ) and $\sum_{n=0}^{\infty} \gamma_{n}=1$.

Proof: Suppose

$$
\begin{aligned}
& f(z)=\sum_{n=0}^{\infty} \gamma_{n} f_{m+n}(z) \\
& =\frac{\gamma_{0}}{z} \\
& +\sum_{n=0}^{\infty} \frac{n^{k}(m+n)(1+\vartheta \theta)+\theta(1-\eta)(1+\vartheta) z^{m+n+1} \gamma_{m+n}}{z\left[n^{k}(m+n)(1+\vartheta \theta)+\theta(1-\eta)(1+\vartheta) z_{0}{ }^{m+n+1}\right]} \\
& \quad=\frac{1}{z}\left[\gamma_{0}\right. \\
& \left.\quad+\sum_{n=0}^{\infty} \frac{n^{k}(m+n)(1+\vartheta \theta) \gamma_{m+n}}{\left.n^{k}(m+n)(1+\vartheta \theta)+\theta(1-\eta)(1+\vartheta) z_{0}{ }^{m+n+1}\right]}\right] \\
& +\sum_{n=0}^{\infty} \frac{\theta(1-\eta)(1+\vartheta) \gamma_{m+n} z^{m+n+1}}{n^{k}(m+n)(1+\vartheta \theta)+\theta(1-\eta)(1+\vartheta) z_{0}{ }^{m+n+1}} . \\
& \operatorname{Then}_{\infty}^{\infty} \text { we have } \\
& \sum_{n=0} \frac{n^{k}(m+n)(1+\vartheta \theta)+\theta(1-\eta)(1+\vartheta) z_{0}{ }^{m+n+1}}{\theta(1-\eta)(1+\vartheta)} \\
& \times \\
& \left(\frac{\theta(1-\eta)(1+\vartheta) \gamma_{m+n}}{n^{k}(m+n)(1+\vartheta \theta)+\theta(1-\eta)(1+\vartheta) z_{0}{ }^{m+n+1}}\right),
\end{aligned}
$$


Now, we have

$$
\sum_{n=0}^{\infty} \gamma_{m+n}=1-\gamma_{0} \leq 1
$$

$$
z_{0} f_{m+n}\left(z_{0}\right)=1
$$

Thus,

$$
z_{0} f\left(z_{0}\right)=\sum_{n=0}^{\infty} \gamma_{m+n} z_{0} f_{m+n}\left(z_{0}\right)=\sum_{n=0}^{\infty} \gamma_{m+n}=1 .
$$

This implies that $f \in \mathfrak{S}_{m 0, k}$.

Therefore $f \in \mathfrak{H}_{m 0, k}^{*}\left(\eta, \theta, \vartheta, z_{0}\right)$.

Conversely, suppose $f \in \mathfrak{H}_{m 0, k}^{*}\left(\eta, \theta, \vartheta, z_{0}\right)$. Since

$$
\begin{gathered}
a_{m+n} \leq \frac{\theta(1-\eta)(1+\vartheta)}{n^{k}(m+n)(1+\vartheta \theta)+\theta(1-\eta)(1+\vartheta) z_{0}{ }^{m+n+1}}, \\
n \geq 0 .
\end{gathered}
$$

Set

$$
=\frac{\left[n^{k}(m+n)(1+\vartheta \theta)+\theta(1-\eta)(1+\vartheta) z_{0}{ }^{m+n+1}\right]}{\theta(1-\eta)(1+\vartheta)} a_{m+n}, n
$$$$
\geq 0 \text {, }
$$$$
\text { and } \gamma_{0}=1-\sum_{n=0}^{\infty} \gamma_{m+n} \text {. }
$$

Then

$$
f(z)=\sum_{n=0}^{\infty} \gamma_{n} f_{n}(z)
$$

This completes the proof of Theorem 4.1.

Theorem 4.2: Let $f_{0}(z)=\frac{1}{z}$,

and

$$
\begin{aligned}
& f_{m+n}(z) \\
& =\frac{n^{k}(m+n)(1+\vartheta \theta)+\theta(1-\eta)(1+\vartheta) z^{m+n+1}}{z(m+n)\left[n^{k}(1+\vartheta \theta)-\theta(1-\eta)(1+\vartheta) z_{0}{ }^{m+n+1}\right]}, \\
& n \geq 0
\end{aligned}
$$

Then $f(z)$ is in the class $\mathfrak{S}_{m 1, k}^{*}\left(\eta, \theta, \vartheta, z_{0}\right)$, if and only if it can be expressed in the form $f(z)=$ $\sum_{n=0}^{\infty} \gamma_{n} f_{n}(z) \quad$ where $\quad \gamma_{n} \geq 0, \gamma_{i}=0(i=$ $1,2, \ldots, m-1, m \geq 2$ ) and $\sum_{n=0}^{\infty} \gamma_{n}=1$.

Corollary 4.1: The extreme points of the class $\mathfrak{S}_{m 0, k}^{*}\left(\eta, \theta, \vartheta, z_{0}\right) \quad$ are the functions $f_{0}(z), f_{m}, f_{m+1}, f_{m+2}, \ldots$ in Theorem 4.1 .

Corollary 4.2: The extreme points of the class $\mathfrak{S}_{m 1, k}^{*}\left(\eta, \theta, \vartheta, z_{0}\right) \quad$ are the functions $f_{0}(z), f_{m}, f_{m+1}, f_{m+2}, \ldots$ in Theorem 4.2.

\section{Closure Theorems}

Theorem 5.1: The class $\mathfrak{H}_{m 0, k}^{*}\left(\eta, \theta, \vartheta, z_{0}\right)$ is closed under convex linear combination

Proof: Suppose that the functions $f, g \in$ $\mathfrak{S}_{m 0, k}^{*}\left(\eta, \theta, \vartheta, z_{0}\right)$ defined by

$$
\begin{gathered}
f(z)=\frac{a_{0}}{z}+\sum_{n=0}^{\infty} a_{m+n} z^{m+n}, a_{0}>0, a_{m+n}>0, z \\
\in \mathcal{U}^{*}
\end{gathered}
$$

and

$$
g(z)=\frac{b_{0}}{z}+\sum_{n=0}^{\infty} b_{m+n} z^{m+n}, b_{0}>0, b_{m+n}>0, z
$$

respectively, it is sufficient to prove that the function $H$ defined by

$$
H(z)=\omega f(z)+(1-\omega) g(z), \quad(0 \leq \omega \leq 1)
$$

is also in the class $\mathfrak{S}_{m 0, k}^{*}\left(\eta, \theta, \vartheta, z_{0}\right)$.

Since

$$
\begin{gathered}
H(z)=\frac{\omega a_{0}+(1-\omega) b_{0}}{z} \\
+\sum_{n=0}^{\infty}\left(\omega a_{m+n}+(1-\omega) b_{m+n}\right) z^{m+n}, a_{0} \\
>0, a_{m+n}>0, z \in \mathcal{U}^{*}
\end{gathered}
$$

we observe that

$$
\begin{aligned}
\sum_{n=0}^{\infty}\left[n^{k}(m+n)(1\right. & +\vartheta \theta) \\
& \left.+z_{0}{ }^{m+n+1}\right]\left(\omega a_{m+n}\right. \\
& \left.+(1-\omega) b_{m+n}\right) \leq \theta(1-\eta)(1 \\
& +\vartheta),
\end{aligned}
$$

with the aid of theorem 2.2.

Thus $H(z) \in \mathfrak{S}_{m 0, k}^{*}\left(\eta, \theta, \vartheta, z_{0}\right)$.

This completes the proof of the theorem.

In a similar manner, by using Theorem 2.3 , we can prove the following theorem.

Theorem 5.2: The class $\mathfrak{S}_{m 1, k}^{*}\left(\eta, \theta, \vartheta, z_{0}\right)$ is closed under convex linear combination.

Proof: The proof is similar to that of Theorem 5.1.

Theorem 5.3: Let the function $f_{l}(z), l=$ $0,1,2, \ldots, q$ defined by

$$
\begin{gathered}
f_{l}(z)=\frac{a_{0, l}}{z}+\sum_{n=0}^{\infty} a_{m+n, l} z^{m+n}, a_{0}>0, a_{m+n, l} \\
>0, z \in \mathcal{U}^{*}
\end{gathered}
$$

be in the class $\mathfrak{H}_{m 0, k}^{*}\left(\eta, \theta, \vartheta, z_{0}\right)$. Then the function

$$
\varphi(z)=\sum_{l=0}^{q} c_{l} f_{l}(z), \quad\left(c_{l} \geq 0\right)
$$

is also in the class $\mathfrak{H}_{m 0, k}^{*}\left(\eta, \theta, \vartheta, z_{0}\right)$, where $\sum_{l=0}^{q} c_{l}=1$. 
Proof: By Theorem 2.2 and for every $l=$ $0,1,2, \ldots, q$ we have

$$
\begin{aligned}
& \sum_{n=0}^{\infty}\left[n^{k}(m+n)(1\right.\left.+\vartheta \theta)+z_{0}^{m+n+1}\right] a_{m+n, l} \\
& \leq \theta(1-\eta)(1+\vartheta)
\end{aligned}
$$

Then,

$$
\begin{aligned}
\varphi(z) & =\sum_{l=0}^{q} c_{l}\left(\frac{a_{0, l}}{z}+\sum_{n=0}^{\infty} a_{m+n, l} z^{m+n}\right), \quad\left(c_{l} \geq 0\right) \\
& =\frac{c_{l} a_{0, l}}{z}+\sum_{n=0}^{\infty}\left(\sum_{l=0}^{q} c_{l} a_{m+n, l}\right) z^{m+n} .
\end{aligned}
$$

Since

$$
\begin{gathered}
\sum_{n=0}^{\infty}\left[n^{k}(m+n)(1+\vartheta \theta)\right. \\
\left.+z_{0}{ }^{m+n+1}\right]\left(\sum_{l=0}^{q} c_{l} a_{m+n, l}\right), \\
=\sum_{l=0}^{q} c_{l}\left(\sum _ { n = 0 } ^ { \infty } \left[n^{k}(m+n)(1+\vartheta \theta)\right.\right. \\
\left.\left.+z_{0}{ }^{m+n+1}\right] a_{m+n, l}\right), \\
\leq\left(\sum_{l=0}^{q} c_{l}\right) \theta(1-\eta)(1+\vartheta), \\
=\theta(1-\eta)(1+\vartheta),
\end{gathered}
$$

Then, $\varphi(z) \in \mathfrak{H}_{m 0, k}^{*}\left(\eta, \theta, \vartheta, z_{0}\right)$.

Theorem 5.4: Let the function $f_{l}(z), l=$ $0,1,2, \ldots, q$ defined by

$$
\begin{gathered}
f_{l}(z)=\frac{a_{0, l}}{z}+\sum_{n=0}^{\infty} a_{m+n, l} z^{m+n}, a_{0}>0, a_{m+n, l} \\
>0, z \in \mathcal{U}^{*}
\end{gathered}
$$

be in the class $\mathfrak{H}_{m 1, k}^{*}\left(\eta, \theta, \vartheta, z_{0}\right)$. Then the function

$$
\varphi(z)=\sum_{l=0}^{q} c_{l} f_{l}(z), \quad\left(c_{l} \geq 0\right)
$$

is also in the class $\mathfrak{H}_{m 1, k}^{*}\left(\eta, \theta, \vartheta, z_{0}\right)$, where $\sum_{l=0}^{q} c_{l}=1$.

Proof: The proof is similar to that of Theorem 5.3.

\section{Convex Family}

Definition 6.1: The family $\mathfrak{H}_{m 0, k}^{*}(\eta, \theta, \vartheta, C)$ is defined by

$$
\mathfrak{S}_{m 0, k}^{*}(\eta, \theta, \vartheta, C)=\cup_{z_{r} \in C} \mathfrak{S}_{m 0, k}^{*}\left(\eta, \theta, \vartheta, z_{r}\right),
$$

where $C$ is a nonempty subset of the real interval $[0,1]$ and $\mathfrak{H}_{m 0, k}^{*}(\eta, \theta, \vartheta, C)$ is defined by a convex family if the subset $C$ consists of one element only by Theorems 5.1 and 5.3.
Now, we have the following results:

Lemma 6.1: Let $z_{1}, z_{2} \in C$ be two distinct positive numbers and $\quad f(z) \in \mathfrak{S}_{m 0, k}^{*}\left(\eta, \theta, \vartheta, z_{0}\right) \cap$ $\mathfrak{S}_{m 0, k}^{*}\left(\eta, \theta, \vartheta, z_{1}\right)$, then $f(z)=\frac{1}{z}$.

Proof: Suppose that $f(z) \in \mathfrak{S}_{m 0, k}^{*}\left(\eta, \theta, \vartheta, z_{1}\right) \cap$ $\mathfrak{H}_{m 0, k}^{*}\left(\eta, \theta, \vartheta, z_{2}\right)$,

we have

$$
\begin{aligned}
a_{0} & =1-\sum_{n=0}^{\infty} a_{m+n} z_{1}{ }^{m+n+1} \\
& =1-\sum_{n=0}^{\infty} a_{m+n} z_{2}{ }^{m+n+1} .
\end{aligned}
$$

Also

$$
\begin{gathered}
f(z)=\frac{a_{0}}{z}+\sum_{n=0}^{\infty} a_{m+n} z^{m+n}, \quad a_{0}>0, a_{m+n} \\
>0, m \in \mathbb{N}
\end{gathered}
$$

Thus, $a_{m+n} \equiv 0, \forall n \geq 0$, because $a_{m+n} \geq 0, z_{1}>0$ and $z_{2}>0$, hence

$$
f(z)=\frac{1}{z}
$$

This completes the proof of the Lemma.

Theorem 6.1: Suppose that $C \subset[0,1]$, then $\mathfrak{S}_{m 0, k}^{*}(\eta, \theta, \vartheta, C)$ is a convex family if and only if $C$ is connected.

Proof: Assume that $C$ is connected and $z_{1}, z_{2} \in C$ with $z_{1}<z_{2}$.

$$
\begin{aligned}
a_{0} & =1-\sum_{n=0}^{\infty} a_{m+n} z_{0}{ }^{m+n+1} \\
& =1-\sum_{n=0}^{\infty} b_{m+n} z_{1}{ }^{m+n+1} .
\end{aligned}
$$

Suppose that the functions $f \in \mathfrak{S}_{m 0, k}^{*}\left(\eta, \theta, \vartheta, z_{0}\right)$ defined by

$$
f(z)=\frac{a_{0}}{z}+\sum_{n=0}^{\infty} a_{m+n} z^{m+n}, a_{0}>0, a_{m+n}>0, z
$$

and $g \in \mathfrak{H}_{m 0, k}^{*}\left(\eta, \theta, \vartheta, z_{1}\right)$

$$
g(z)=\frac{b_{0}}{z}+\sum_{n=0}^{\infty} b_{m+n} z^{m+n}, b_{0}>0, b_{m+n}>0, z
$$

$\in \mathcal{U}^{*}$

it is sufficient to prove that the function $H$ defined by

$$
H(z)=\omega f(z)+(1-\omega) g(z), \quad(0 \leq \omega \leq 1)
$$
that there exists a $z_{2}\left(z_{0} \leq z_{2} \leq z_{1}\right)$ is also in the class $\mathfrak{H}_{m 0, k}^{*}\left(\eta, \theta, \vartheta, z_{2}\right)$. 
Then

$$
\begin{gathered}
K(z)=z H(z) \\
K(z)=\omega a_{0}+(1-\omega) b_{0} \\
+\sum_{n=0}^{\infty}\left(\omega a_{m+n}+(1-\omega) b_{m+n}\right) z^{m+n}, a_{0} \\
>0, a_{m+n}>0, z \in U^{*} \\
+\omega \sum_{n=0}^{\infty}\left(z^{m+n}-z_{0}^{m+n}\right) a_{m+n} \\
+(1-\omega) \sum_{n=0}^{\infty}\left(z^{m+n}-z_{1}^{m+n}\right) b_{m+n}, a_{0}>0, a_{m+n} \\
>0, z \in U^{*}
\end{gathered}
$$

since $z$ is real number, then $K(z)$ is also real number also we have

$K\left(z_{0}\right) \leq 1$ and $K\left(z_{1}\right) \geq 1$, there exists $z_{2} \in\left[z_{0}, z_{1}\right]$, such that $K\left(z_{2}\right)=1$.

Therefore,

$$
z_{2} H\left(z_{2}\right)=z_{2}, \quad\left(z_{0} \leq z_{2} \leq z_{1}\right)
$$

this implies that

$$
H(z) \in \mathfrak{H}_{m 0, k}^{*}
$$

We observe that

$$
\begin{aligned}
& \sum_{n=0}^{\infty}\left[n^{k}(m+n)(1+\vartheta \theta)+z_{2}{ }^{m+n+1}\right]\left(\omega a_{m+n}+\right. \\
& \left.(1-\omega) b_{m+n}\right) \\
& =\omega \sum_{n=0}^{\infty}\left[n^{k}(m+n)(1+\vartheta \theta)+z_{0}{ }^{m+n+1}\right] a_{m+n} \\
& +(1-\omega) \sum_{n=0}^{\infty}\left[n^{k}(m+n)(1+\vartheta \theta)\right. \\
& \left.+z_{1}^{m+n+1}\right] b_{m+n} \\
& +\theta(1-\eta)(1 \\
& +\vartheta) \omega \sum_{n=0}^{\infty}\left(z_{2}{ }^{m+n+1}-z_{0}{ }^{m+n+1}\right) a_{m+n} \\
& +\theta(1-\eta)(1+\vartheta)(1 \\
& -\omega) \sum_{n=0}^{\infty}\left(z_{2}{ }^{m+n+1}-z_{1}{ }^{m+n+1}\right) b_{m+n} \\
& =\omega \sum_{n=0}^{\infty}\left[n^{k}(m+n)(1+\vartheta \theta)+z_{0}{ }^{m+n+1}\right] a_{m+n} \\
& +(1-\omega) \sum_{n=0}^{\infty}\left[n^{k}(m+n)(1+\vartheta \theta)\right. \\
& \left.+z_{1}^{m+n+1}\right] b_{m+n} \\
& \leq \theta(1-\eta)(1+\vartheta)+(1-\omega) \theta(1-\eta)(1+\vartheta) \\
& =\theta(1-\eta)(1+\vartheta) \text {. }
\end{aligned}
$$

With the aid of theorem 2.2.

Thus, $H(z) \in \mathfrak{H}_{m 0, k}^{*}\left(\eta, \theta, \vartheta, z_{2}\right)$.

Since $z_{1}$ and $z_{2}$ are arbitrary numbers, the family $\mathfrak{H}_{m 0, k}^{*}(\eta, \theta, \vartheta, C)$ is convex.

Conversely, if the set $C$ is not connected, then there exists $z_{0}, z_{1}$ and $z_{2}$ such that $z_{0}, z_{1} \in C$ and $z_{2} \notin C$ and $z_{0}<z_{2}<z_{1}$.

Now, let $f(z) \in \mathfrak{H}_{m 0, k}^{*}\left(\eta, \theta, \vartheta, z_{0}\right)$, and $g(z) \in$ $\mathfrak{S}_{m 0, k}^{*}\left(\eta, \theta, \vartheta, z_{1}\right)$

Therefore,

$$
\begin{gathered}
K(\omega)=K\left(z_{2}, \omega\right) \\
=1+\omega \sum_{n=0}^{\infty}\left(z_{2}{ }^{m+n+1}-z_{0}{ }^{m+n+1}\right) a_{m+n} \\
+(1-\omega) \sum_{n=0}^{\infty}\left(z_{2}{ }^{m+n+1}-z_{1}{ }^{m+n+1}\right) b_{m+n}, a_{0} \\
>>0, a_{m+n}>0, z \in \mathcal{U}^{*}
\end{gathered}
$$

for fixed $z_{2}$ and $0 \leq \omega \leq 1$.

Since $K\left(z_{2}, 0\right)<1$ and $K\left(z_{2}, 1\right)>1$, there exists $\omega_{0} ; 0<\omega_{0}<1$, such that $K\left(z_{2}, \omega_{0}\right)=1$ or $z_{2} K\left(z_{2}\right)=1$,

where $K(z)=\omega_{0} f(z)+\left(1-\omega_{0}\right) g(z)$.

Therefore $K(z) \in \mathfrak{H}_{m 0, k}^{*}\left(\eta, \theta, \vartheta, z_{0}\right)$

Also $K(z) \notin \mathfrak{H}_{m 0, k}^{*}(\eta, \theta, \vartheta, C)$ using Lemma 6.1.

Since $z_{2} \in C$ and $K(z) \neq z$.

Thus the family $\mathfrak{H}_{m 0, k}^{*}(\eta, \theta, \vartheta, C)$ is not convex which is a contradiction.

This completes the proof of theorem.

Conclusion: The main impact of this paper is to is to introduce a new subclasses of meromorphic univalent functions, and study their geometrical properties, like coefficient estimate, distortion theorem, extreme points and convex family.

\section{References}

[1] M. K. Aouf, A certain subclass of meromorphically starlike functions with positive coefficients, Rend. Mat., 9(1989), 225-235.

[2] M. K. Aouf, On a certain class of meromorphically univalent functions with positive coefficients, Rend. Mat., 11(1991), 209-219.

[3] M. K. Aouf and H. E. Darwish, On meromorphic univalent functions with positive coefficients and fixed two points, Ann. St. Univ. A. I. Cuza, Iasi, Tomul XLII, Matem., (1996), 3-14.

[4] W. G. Atshan, Subclass of meromorphic functions with positive coefficients defined by Ruscheweyh derivative II, Surveys in Math. and its Appl., 3(2008), 67-77. 
[5] S. K. Bajpai, A note on a class of meromorphic univalent functions, Rev. Roum. Math. Pures App., 22(1977), 295-297.

[6] N. E. Cho, On certain class of meromorphic functions with positive coefficients, J. Inst. Math. Comput. Sci., 3,2(1990), 119-125.

[7] N. E. Cho, K. Inayatnoor, Inclusion properties for certain classes of meromorphic functions associated with the Choi-Saigo-Srivastava operator, J. Math. Anal. Appl., 320(2006), 779-786.

[8] N. E. Cho, I. H. Kim, Inclusion properties for certain classes of meromorphic functions associated with the generalized hypergeometric function, Applied Mathematics and Computation, 187(2007), 115-121.

[9] R. M. El- Ashwah, M. K. Aouf, Hadamard product of certain meromorphic starllike and convex functions, Comput. Math. Appl., 57(2009), 11021106.

[10] B. A. Frasin, M. Darus, On certain meromorphic functions with positive coefficients, Southeast Asian Bull. Math. , 28(2004), 615-623.

[11] F. Ghanim, M. Darus, On certain subclass of merpmorphic univalent functions with fixed residue, Far East J. Math. Sci., 26(2007), 195-207.

[12] F. Ghanim, M. Darus, A new subclass on uniformly starlike and convex functions with negative coefficients II, Int. J. Pure Appl. Math., 45, 4(2009), 559-572.

[13] F. Ghanim, M. Darus, On a certain subclass of meromorphic univalent functions with fixed second positive coefficients, Surveys in Mathematics and its Applications, 5(2010), 49-60.
[14] S. B. Joshi, S. R. Kulkarni and N. K. Thakare, Subclasses of meromorphic functions with missing coefficients, J. Analysis, 2(1994), 23-29.

[15] Krzysztof, Piejko, J. Sokol, Subclasses of meromorphic functions associated with the ChoKnow-Srivastava operator, J. Math. Anal. Appl., 337(2008), 1261-1266.

[16] J. E. Miller, Convex meromorphic mapping and related functions, Proc. Amer. Math. Soc., 25(1970), 220-222.

[17] M. L. Mogra, T. R. Reddy, O. P. Juneja, Meromorphic univalent functions with positive coefficients, Bull. Austral. Math. Soc., 32(1985), 161-176.

[18] S. Mouyuan, Z. Mingliu, H. M. Srivastava, Some inclusion relationships and integral preserving properties of certain subclasses of meromprphic functions associated with a family of integral operators, J. Math. Anal. Appl., 337(2008), 505-515. [19] C. Pommerenke, On meromorphic starlike functions, Pacific J. Math., 13(1963), 221-235.

[20] W. C. Poyster, Meromorphic starlike multivalent functions, Trans. Am. Math. Soc. 107(1963), 300-308.

[21] H. M. Srivastava, S. Owa, Current topics in analytic functions theory, World Scientific, Singapore, 1992.

[22] B. A. Uralegaddi and M. D. Ganigi, Meromprphic starlike functions with two fixed points, Bull. Iranian Math. Soc., 14, No.1(1987), 1021.

[23] B.A. Uralegaddi, C. Somanatha, New criteria for meromorphic starlike univalent functions, Bull. Austral. Math. Soc., 43(1991), 137-140. 
حول اصناف جديدة من الدوال احادية التكافؤ الميرومورفية بمؤثر تفاضلي

$$
\text { هازه زرار حسين }
$$

قسم الرياضيات، كلية العلوم، جامعة صلاح الدين، اربيل، عراق

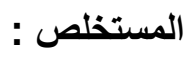

في هذا البحث نعرف وندرس اصناف جديدة (البر الميرومورفية المعرفة بواسطة مؤثر تفاضلي ، ونحصل على العديد من النتائج المهمة مثل متباينة المعاملات، و النقاط القصوى، نظرية البعد، التركيب المحدب للاصناف من الدو ال السابقة. 